\title{
A Note on the Fractional Generalized Higher Order KdV Equation
}

\author{
Yongyi Gu \\ School of Statistics and Mathematics, Guangdong University of Finance and Economics, Guangzhou 510320, China \\ Correspondence should be addressed to Yongyi Gu; gdguyongyi@163.com
}

Received 30 May 2018; Accepted 19 July 2018; Published 23 August 2018

Academic Editor: Xinguang Zhang

Copyright (C) 2018 Yongyi Gu. This is an open access article distributed under the Creative Commons Attribution License, which permits unrestricted use, distribution, and reproduction in any medium, provided the original work is properly cited.

We obtain exact solutions to the fractional generalized higher order Korteweg-de Vries (KdV) equation using the complex method. It has showed that the applied method is very useful and is practically well suited for the nonlinear differential equations, those arising in mathematical physics.

\section{Introduction}

Nonlinear fractional differential equations (NFDEs) are universally applied in signal processing, electrical networks, acoustics, fluid dynamics, biology, chemistry, physics, etc. For example, the singular behaviours [1-9] and impulsive phenomena [10-19] often exhibit some blow-up properties [20-25] which occur in a lot of complex physical processes. NFDEs have been attracted extensive attention and have been widely investigated [26-38]. Exact solutions of NFDEs play an important role in the study of mathematical physics phenomena. Therefore, seeking exact solutions of NFDEs is an interesting and significant subject.

The fractional generalized higher order $\mathrm{KdV}$ equation is a useful model. Applying the generalized $\exp (-\Phi(\zeta))$ expansion method, Lu et al. [39] obtained exact solutions of this equation. In this article, we would like to utilize the complex method [40-43] to seek exact solutions to the fractional generalized higher order $\mathrm{KdV}$ equation.

\section{Preliminaries}

Let $f: R \longrightarrow R, x \longrightarrow f(x)$ be a continuous function and $v>0$ denote a constant discrete span. Define the operator $F W(\nu)$ as follows:

$$
F W(\nu) f(x):=f(x+\nu),
$$

then the fractional difference of $f(x)$ of order $\mu$ can be expressed as

$$
\begin{aligned}
\Delta^{\mu} f(x) & =(F W-1)^{\mu} f(x) \\
& =\sum_{n=0}^{\infty}(-1)^{n}\left(\begin{array}{l}
\mu \\
n
\end{array}\right) f[x+(\mu-n) \nu],
\end{aligned}
$$

where $0<\mu \leq 1$, and its fractional derivative of order $\mu$ can be expressed as

$$
f^{(\mu)}(x)=\lim _{\nu \longrightarrow 0}\left(\frac{\sum_{n=0}^{\infty}(-1)^{n}\left(\begin{array}{c}
\mu \\
n
\end{array}\right) f[x+(\mu-n) \nu]}{\nu^{\mu}}\right) .
$$

The above is expressed as

$$
\begin{array}{r}
\frac{1}{\Gamma(-\mu)} \int_{0}^{x}(x-z)^{-\mu-1} f(z) d z, \quad \mu<0, \\
\frac{1}{\Gamma(1-\mu)} \frac{d}{d_{x}} \int_{0}^{x}(x-z)^{-\mu}[f(z)-f(0)] d z,
\end{array}
$$

$$
0<\mu<1,
$$

$$
\left(f^{(\mu-n)}(x)\right)^{(n)}, \quad n \leq \mu \leq n+1, n \geq 1 .
$$

Further, Jumarie's modified Riemann-Liouville derivative $[44,45]$ is given by

$$
D_{x}^{\mu} x^{\gamma}=\frac{\Gamma(1+\gamma)}{\Gamma(1+\gamma-\mu)} x^{(\gamma-\mu)}, \quad \gamma>0,
$$


then its related NFDE is given by

$$
\begin{aligned}
f\left(u, u_{t}, u_{x}, D_{t}^{\mu} u, D_{x}^{\mu} u, D_{t}^{\gamma} u, D_{x}^{\gamma} u, \ldots\right)=0, & \\
& 0<\mu \leq 1 .
\end{aligned}
$$

Let $m \in \mathbb{N}:=\{1,2,3, \ldots\}, r_{j} \in\{0,1,2, \ldots\}, j=0,1, \ldots, m$, $r=\left(r_{0}, r_{1}, \ldots, r_{m}\right)$, and

$$
K_{r}[w](z):=\prod_{j=0}^{m}\left[w^{(j)}(z)\right]^{r_{j}},
$$

then the degree of $K_{r}[w]$ is denoted by $d(r):=\sum_{j=0}^{m} r_{j}$. We define the differential polynomial as

$$
F\left(w, w^{\prime}, \ldots, w^{(m)}\right):=\sum_{r \in H} a_{r} K_{r}[w]
$$

in which $H$ is a finite index set and $a_{r}$ are constants. The degree of $F\left(w, w^{\prime}, \ldots, w^{(m)}\right)$ can be denoted by $\operatorname{deg} F\left(w, w^{\prime}, \ldots, w^{(m)}\right):=\max _{r \in H}\{d(r)\}$.

The ordinary differential equation (ODE) is given by

$$
F\left(w, w^{\prime}, \ldots, w^{(m)}\right)=c w^{n}+d,
$$

where $c \neq 0, d$ are constants, $n \in \mathbb{N}$.

Suppose that the meromorphic solutions $w$ of (9) have at least one pole. Let $p, q \in \mathbb{N}$ and insert the Laurent series

$$
w(z)=\sum_{\tau=-q}^{\infty} \beta_{\tau} z^{\tau}, \quad \beta_{-q} \neq 0, q>0,
$$

into (9); if it is determined $p$ different Laurent singular parts:

$$
\sum_{\tau=-q}^{-1} \beta_{\tau} z^{\tau}
$$

then (9) is said to satisfy the weak $\langle p, q\rangle$ condition.

Give two complex numbers $v_{1}, v_{2}$ such that $\operatorname{Im}\left(\nu_{1} / v_{2}\right)>$ 0 , let $L$ be the discrete subset $L\left[2 v_{1}, 2 v_{2}\right]:=\left\{v \mid v=2 c_{1} v_{1}+\right.$ $\left.2 c_{2} v_{2}, c_{1}, c_{2} \in \mathbb{Z}\right\}$, and $\mathrm{L}$ is isomorphic to $\mathbb{Z} \times \mathbb{Z}$. Let the discriminant $\Delta=\Delta\left(b_{1}, b_{2}\right):=b_{1}^{3}-27 b_{2}^{2}$ and

$$
h_{n}:=h_{n}(L):=\sum_{\nu \in L \backslash\{0\}} \frac{1}{v^{n}} .
$$

A meromorphic function $\wp(z):=\wp\left(z, g_{2}, g_{3}\right)$ with double periods $2 v_{1}, 2 v_{2}$, which satisfies the equation

$$
\left(\wp^{\prime}(z)\right)^{2}=4 \wp^{3}(z)-g_{2} \wp(z)-g_{3}
$$

in which $\Delta\left(g_{2}, g_{3}\right) \neq 0, g_{2}=60 h_{4}$, and $g_{3}=140 h_{6}$, is called the Weierstrass elliptic function.

If a meromorphic function $\varsigma$ is a rational function of $z$, an elliptic function, or a rational function of $e^{\alpha z}, \alpha \in \mathbb{C}$, then we say that $\varsigma$ belongs to the class $W$.

In 2009, Eremenko et al. [46] considered the following $m$-order Briot-Bouquet equation (BBEq):

$$
F\left(w, w^{(m)}\right)=\sum_{j=0}^{n} F_{j}(w)\left(w^{(m)}\right)^{j}=0,
$$

where $m \in \mathbb{N}$ and $F_{j}(w)$ are constant coefficient polynomials. For the $m$-order BBEq, we have the lemma as follows.
Lemma 1 (see $[41,47,48])$. Let $m, s, n, p \in \mathbb{N}$, and $\operatorname{deg} F\left(w, w^{(m)}\right)<n$, and the $m$-order BBEq

$$
F\left(w, w^{(m)}\right)=c w^{n}+d
$$

satisfies weak $\langle p, q\rangle$ condition, then the meromorphic solutions $w(z)$ belong to the class $W$. Suppose that for some values of parameters such solutions $w$ exist, then other meromorphic solutions should form one-parametric family $\left(z-z_{0}\right), z_{0} \in \mathbb{C}$. Then each elliptic solution with a pole at $z=0$ can be expressed as

$$
\begin{aligned}
w(z)= & \sum_{i=1}^{s-1} \sum_{j=2}^{q} \frac{(-1)^{j} \beta_{-i j}}{(j-1) !} \frac{d^{j-2}}{d z^{j-2}} \\
& \cdot\left(\frac{1}{4}\left[\frac{\wp^{\prime}(z)+C_{i}}{\wp(z)-D_{i}}\right]^{2}-\wp(z)\right)+\sum_{i=1}^{s-1} \frac{\beta_{-i 1}}{2} \\
& \cdot \frac{\wp^{\prime}(z)+C_{i}}{\wp(z)-D_{i}}+\sum_{j=2}^{q} \frac{(-1)^{j} \beta_{-s j}}{(j-1) !} \frac{d^{j-2}}{d z^{j-2}} \wp(z) \\
& +\beta_{0},
\end{aligned}
$$

in which $\beta_{-i j}$ are determined by (10), $\sum_{i=1}^{s} \beta_{-i 1}=0$, and $C_{i}^{2}=$ $4 D_{i}^{3}-g_{2} D_{i}-g_{3}$.

Each rational function solution $R(z)$ can be expressed as

$$
R(z)=\sum_{i=1}^{s} \sum_{j=1}^{q} \frac{\beta_{i j}}{\left(z-z_{i}\right)^{j}}+\beta_{0},
$$

and it has $s(\leq p)$ distinct poles of multiplicity $q$.

Each simply periodic solution $R(\eta)$ is a rational function of $\eta=e^{\alpha z}(\alpha \in \mathbb{C})$ and can be expressed as

$$
R(\eta)=\sum_{i=1}^{s} \sum_{j=1}^{q} \frac{\beta_{i j}}{\left(\eta-\eta_{i}\right)^{j}}+\beta_{0}
$$

and it has $s(\leq p)$ distinct poles of multiplicity $q$.

Lemma 2 (see [48, 49]). Weierstrass elliptic functions $\wp(z)$ have the following addition formula:

$$
\begin{aligned}
\wp\left(z-z_{0}\right)= & -\wp\left(z_{0}\right)-\wp(z) \\
& +\frac{1}{4}\left[\frac{\wp^{\prime}(z)+\wp^{\prime}\left(z_{0}\right)}{\wp(z)-\wp\left(z_{0}\right)}\right]^{2} .
\end{aligned}
$$

When $g_{2}=g_{3}=0$, it can be degenerated to rational functions according to

$$
\wp(z, 0,0)=\frac{1}{z^{2}}
$$

When $\Delta\left(g_{2}, g_{3}\right)=0$, it can also be degenerated to simple periodic functions according to

$$
\wp\left(z, 3 d^{2},-d^{3}\right)=2 d-\frac{3 d}{2} \operatorname{coth}^{2} \sqrt{\frac{3 d}{2}} z .
$$




\section{Main Results}

The fractional generalized higher order $\mathrm{KdV}$ equation [39] is given as

$$
D_{t}^{\mu}+u u_{x}-u u_{x x x}+u_{x x x x x}=0
$$

Substituting traveling wave transform

$$
u(x, t)=w(z), \quad z=k\left(x+\frac{\lambda t^{\mu}}{\Gamma(1+\mu)}\right),
$$

into (22), we get

$$
k \lambda w^{\prime}+k w w^{\prime}-k^{3} w w^{\prime \prime \prime}+k^{5} w^{(5)}=0,
$$

Integrating (24) yields

$$
\lambda w+\frac{w^{2}}{2}+k^{2} w w^{\prime \prime}-\frac{k^{2}}{2}\left(w^{\prime}\right)^{2}+k^{4} w^{(4)}+\delta=0,
$$

where $k$ and $\lambda$ are constants and $\delta$ is the integral constant.

Theorem 3. If $k \neq 0$, then the meromorphic solutions $w(z)$ of (25) have the following forms.

(I) The rational function solutions

$$
w_{r}(z)=\frac{-30 k^{2}}{\left(z-z_{0}\right)^{2}}+\frac{5}{2},
$$

where $\lambda=-5 / 2, \delta=25 / 8$, and $z_{0} \in \mathbb{C}$.

(II) The simply periodic solutions

$$
w_{s}(z)=-\frac{15 k^{2} \alpha^{2}}{2} \operatorname{coth}^{2} \frac{\alpha\left(z-z_{0}\right)}{2}+\frac{10 k^{2} \alpha^{2}+5}{2}
$$

where $\lambda=\left(3 k^{4} \alpha^{4}-5\right) / 2, \delta=\left(30 k^{6} \alpha^{6}-55 k^{4} \alpha^{4}+25\right) / 8$, and $z_{0} \in \mathbb{C}$.

(III) The elliptic function solutions

$$
\begin{aligned}
w_{d}(z)= & -30 k^{2}\left(-\wp(z)+\frac{1}{4}\left(\frac{\wp^{\prime}(z)+D}{\wp(z)-C}\right)^{2}\right) \\
& +30 k^{2} C+\frac{5}{2}
\end{aligned}
$$

where $C^{2}=4 D^{3}-g_{2} D-g_{3}, g_{2}=(2 \lambda+5) / 36 k^{4}$, and $g_{3}=$ $-(100+55 \lambda+12 \delta) / 9720 k^{6}$.

Proof. Substituting (10) into (25) we have $p=1, q=2, \beta_{-2}=$ $-30 k^{2}, \beta_{-1}=0, \beta_{0}=5 / 2, \beta_{1}=0, \beta_{2}=-(2 \lambda+5) / 24 k^{2}$, and $\beta_{3}$ is an arbitrary constant.

Therefore, $(25)$ satisfies the weak $\langle 1,2\rangle$ condition. In the following, we will show the meromorphic solutions of (25).

By (17), we infer that the indeterminant rational solutions of (25) are

$$
R_{1}(z)=\frac{\beta_{11}}{z^{2}}+\frac{\beta_{12}}{z}+\beta_{10}
$$

with pole at $z=0$.
Inserting $R_{1}(z)$ into (25), we have

$$
\begin{aligned}
\frac{1}{2} \beta_{10}{ }^{2} & +\lambda \beta_{10}+\delta+\frac{\lambda \beta_{11}+\beta_{11} \beta_{10}}{z} \\
& +\frac{2 \beta_{12} \beta_{10}+\beta_{11}^{2}+2 \lambda \beta_{12}}{2 z^{2}} \\
& +\frac{2 k^{2} \beta_{10} \beta_{11}+\beta_{12} \beta_{11}}{z^{3}} \\
& +\frac{3 k^{2} \beta_{11}^{2}+12 k^{2} \beta_{10} \beta_{12}+\beta_{12}^{2}}{2 z^{4}} \\
& +\frac{24 k^{4} \beta_{11}+6 k^{2} \beta_{11} \beta_{12}}{z^{5}}+\frac{120 k^{4} \beta_{12}+4 k^{2} \beta_{12}^{2}}{z^{6}} \\
= & 0,
\end{aligned}
$$

then we get $\beta_{12}=-30 k^{2}, \beta_{11}=0$, and $\beta_{10}=5 / 2$.

So we can determine that

$$
R_{1}(z)=\frac{-30 k^{2}}{z^{2}}+\frac{5}{2}
$$

where $\lambda=-5 / 2$ and $\delta=25 / 8$.

Therefore the rational solutions of (25) are

$$
w_{r}(z)=\frac{-30 k^{2}}{\left(z-z_{0}\right)^{2}}+\frac{5}{2},
$$

where $\lambda=-5 / 2, \delta=25 / 8$, and $z_{0} \in \mathbb{C}$.

Let $\eta=e^{\alpha z}$. To derive simply periodic solutions, we substitute $w=R(\eta)$ into (25) to yield

$$
\begin{gathered}
k^{4} \alpha^{4}\left(R^{(4)} \eta^{4}+6 R^{\prime \prime \prime} \eta^{3}+7 R^{\prime \prime} \eta^{2}+R^{\prime} \eta\right)-\frac{k^{2}}{2}\left(\alpha R^{\prime} \eta\right)^{2} \\
+k^{2} \alpha^{2} R\left(\eta R^{\prime}+\eta^{2} R^{\prime \prime}\right)+\frac{R^{2}}{2}+\lambda R+\delta=0
\end{gathered}
$$

Substituting

$$
R_{2}(z)=\frac{\beta_{21}}{(\eta-1)^{2}}+\frac{\beta_{22}}{(\eta-1)}+\beta_{20}
$$

into (33), we obtain that

$$
R_{2}(z)=-\frac{30 k^{2} \alpha^{2}}{(\eta-1)^{2}}-\frac{30 k^{2} \alpha^{2}}{(\eta-1)}-\frac{5 k^{2} \alpha^{2}}{2}+\frac{5}{2},
$$

where $\lambda=\left(3 k^{4} \alpha^{4}-5\right) / 2$ and $\delta=\left(30 k^{6} \alpha^{6}-55 k^{4} \alpha^{4}+25\right) / 8$.

Substituting $\eta=e^{\alpha z}$ into (35), we achieve simply periodic solutions to (25) with pole at $z=0$

$$
\begin{aligned}
w_{s 0}(z) & =-\frac{30 k^{2} \alpha^{2}}{\left(e^{\alpha z}-1\right)^{2}}-\frac{30 k^{2} \alpha^{2}}{\left(e^{\alpha z}-1\right)}-\frac{5 k^{2} \alpha^{2}}{2}+\frac{5}{2} \\
& =-30 k^{2} \alpha^{2} \frac{e^{\alpha z}}{\left(e^{\alpha z}-1\right)^{2}}-\frac{5 k^{2} \alpha^{2}}{2}+\frac{5}{2} \\
& =-\frac{15 k^{2} \alpha^{2}}{2} \operatorname{coth}^{2} \frac{\alpha z}{2}+\frac{10 k^{2} \alpha^{2}+5}{2},
\end{aligned}
$$

where $\lambda=\left(3 k^{4} \alpha^{4}-5\right) / 2$ and $\delta=\left(30 k^{6} \alpha^{6}-55 k^{4} \alpha^{4}+25\right) / 8$. 
Thurs, simply periodic solutions of (25) are

$$
w_{s}(z)=-\frac{15 k^{2} \alpha^{2}}{2} \operatorname{coth}^{2} \frac{\alpha\left(z-z_{0}\right)}{2}+\frac{10 k^{2} \alpha^{2}+5}{2},
$$

where $\lambda=\left(3 k^{4} \alpha^{4}-5\right) / 2, \delta=\left(30 k^{6} \alpha^{6}-55 k^{4} \alpha^{4}+25\right) / 8$, and $z_{0} \in \mathbb{C}$.

From (16) of Lemma 1, the elliptic solutions of (25) are expressed as

$$
w_{d 0}(z)=\beta_{-2 \wp} \wp(z)+\beta_{30},
$$

with pole at $z=0$.

Putting $w_{d 0}(z)$ into (25), we get

$$
w_{d 0}(z)=-30 k^{2} \wp(z)+\frac{5}{2},
$$

where $g_{2}=(2 \lambda+5) / 36 k^{4}$ and $g_{3}=-(100+55 \lambda+12 \delta) / 9720 k^{6}$.

So, the elliptic solutions of (25) are

$$
w_{d}(z)=-30 k^{2} \wp\left(z-z_{0}\right)+\frac{5}{2},
$$

where $z_{0} \in \mathbb{C}$.

We can apply the addition formula to rewrite it as

$$
\begin{aligned}
w_{d}(z)= & -30 k^{2}\left(-\wp(z)+\frac{1}{4}\left(\frac{\wp^{\prime}(z)+D}{\wp(z)-C}\right)^{2}\right) \\
& +30 k^{2} C+\frac{5}{2},
\end{aligned}
$$

where $C^{2}=4 D^{3}-g_{2} D-g_{3}, g_{2}=(2 \lambda+5) / 36 k^{4}$, and $g_{3}=$ $-(100+55 \lambda+12 \delta) / 9720 k^{6}$.

Substitute traveling wave transform into the meromorphic solutions $w(z)$ of (25) to get traveling wave exact solutions to the fractional generalized higher order $\mathrm{KdV}$ equation. So we obtain Theorem 4 as follows.

Theorem 4. If $k \neq 0$, then traveling wave solutions of (25) have the following forms.

(I) The rational function solutions

$$
w_{r}(x, t)=w_{r}\left(k x-\frac{5 k t^{\mu}}{2 \Gamma(1+\mu)}\right),
$$

where $\lambda=-5 / 2, \delta=25 / 8$, and $z_{0} \in \mathbb{C}$.

(II) The simply periodic solutions

$$
w_{s}(x, t)=w_{s}\left(k x+\frac{\left(3 k^{5} \alpha^{4}-5 k\right) t^{\mu}}{2 \Gamma(1+\mu)}\right),
$$

where $\lambda=\left(3 k^{4} \alpha^{4}-5\right) / 2, \delta=\left(30 k^{6} \alpha^{6}-55 k^{4} \alpha^{4}+25\right) / 8$, and $z_{0} \in \mathbb{C}$.

(III) The elliptic function solutions

$$
w_{d}(x, t)=w_{d}\left(k x+\frac{k \lambda t^{\mu}}{\Gamma(1+\mu)}\right),
$$

where $C^{2}=4 D^{3}-g_{2} D-g_{3}, g_{2}=(2 \lambda+5) / 36 k^{4}$, and $g_{3}=$ $-(100+55 \lambda+12 \delta) / 9720 k^{6}$.

\section{Conclusions}

In this note, we have used the complex method to construct exact solutions to the mentioned NFDE. Although we do not show that the meromorphic solutions of the fractional generalized higher order KdV equation belong to the class $W$, we can still obtain the meromorphic solutions to this NFDE and then get its traveling wave exact solutions. The results demonstrate that the applied method is direct and efficient method, which allows us to do tedious and complicated algebraic calculation. We can utilize these ideas to other NFDEs.

\section{Data Availability}

No data were used to support this study.

\section{Conflicts of Interest}

The authors declare that they have no conflicts of interest.

\section{Acknowledgments}

This work was supported by the NSF of China (11701111 and 11271090); the NSF of Guangdong Province (2016A030310257); Guangdong Universities (Basic and Applied Research) Major Project (2017KZDXM038); Guangzhou City Social Science Federation "Yangcheng Young Scholars” Project (18QNXR35).

\section{References}

[1] X. Hao, L. Liu, and Y. Wu, "On positive solutions of m-point nonhomogeneous singular boundary value problem," Nonlinear Analysis, vol. 73, no. 8, pp. 2532-2540, 2010.

[2] X. Zhang and L. Liu, "A necessary and sufficient condition of positive solutions for nonlinear singular differential systems with four-point boundary conditions," Applied Mathematics and Computation, vol. 215, no. 10, pp. 3501-3508, 2010.

[3] P. Li and G. Ren, "Some classes of equations of discrete type with harmonic singular operator and convolution," Applied Mathematics and Computation, vol. 284, pp. 185-194, 2016.

[4] Y. Guan, Z. Zhao, and X. Lin, "On the existence of positive solutions and negative solutions of singular fractional differential equations via global bifurcation techniques," Boundary Value Problems, vol. 2016, no. 1, article 141, 2016.

[5] X. Hao, "Positive solution for singular fractional differential equations involving derivatives," Advances in Difference Equations, Article ID 139, 2016.

[6] Z. Zheng and Q. Kong, "Friedrichs extensions for singular Hamiltonian operators with intermediate deficiency indices," Journal of Mathematical Analysis and Applications, vol. 461, no. 2, pp. 1672-1685, 2018.

[7] F. Yan, M. Zuo, and X. Hao, "Positive solution for a fractional singular boundary value problem with p-Laplacian operator," Boundary Value Problems, article no. 51, 10 pages, 2018.

[8] J. Jiang, L. Liu, and Y. Wu, "Positive solutions to singular fractional differential system with coupled boundary conditions," Communications in Nonlinear Science and Numerical Simulation, vol. 18, no. 11, pp. 3061-3074, 2013. 
[9] J. Liu and Z. Zhao, "Existence of positive solutions to a singular boundary-value problem using variational methods," Electronic Journal of Differential Equations, vol. 2014, no. 135, 9 pages, 2014.

[10] X. Hao, L. Liu, and Y. Wu, "Positive solutions for second order impulsive differential equations with integral boundary conditions," Communications in Nonlinear Science and Numerical Simulation, vol. 16, no. 1, pp. 101-111, 2011.

[11] X. Hao, M. Zuo, and L. Liu, "Multiple positive solutions for a system of impulsive integral boundary value problems with sign-changing nonlinearities," Applied Mathematics Letters, vol. 82, pp. 24-31, 2018.

[12] J. Wang, A. G. Ibrahim, and D. O’Regan, “Topological structure of the solution set for fractional non-instantaneous impulsive evolution inclusions," Journal of Fixed Point Theory and Applications, vol. 20, no. 2, Art. 59, 25 pages, 2018.

[13] Y. M. Xu and H. J. Zhang, "Positive solutions of an infnite boundary value problem for nth-order nonlinear impulsive singular integro-differential equations in Banach spaces," Applied Mathematics and Computation, vol. 218, no. 9, pp. 5806-5818, 2012.

[14] J. Liu and Z. Zhao, "An application of variational methods to second-order impulsive differential equation with derivative dependence," Electronic Journal of Differential Equations, vol. 2014, no. 62, pp. 1-13, 2014.

[15] L. Gao, D. Wang, and G. Wang, "Further results on exponential stability for impulsive switched nonlinear time-delay systems with delayed impulse effects," Applied Mathematics and Computation, vol. 268, pp. 186-200, 2015.

[16] X. Hao and L. Liu, "Mild solution of semilinear impulsive integro-differential evolution equation in Banach spaces," Mathematical Methods in the Applied Sciences, vol. 40, no. 13, pp. 4832-4841, 2017.

[17] Y. L. Guan, Z. Q. Zhao, and X. L. Lin, "On the Existence of Solutions for Impulsive Fractional Differential Equations," Advances in Mathematical Physics, vol. 2017, Article ID 1207456, 12 pages, 2017.

[18] Y. Wang and Z. Zhao, "Existence and multiplicity of solutions for a second-order impulsive differential equation via variational methods," Advances in Difference Equations, Paper No. 46, 9 pages, 2017.

[19] J. Liu and Z. Zhao, "Multiple solutions for impulsive problems with non-autonomous perturbations," Applied Mathematics Letters, vol. 64, pp. 143-149, 2017.

[20] Q. Gao, F. Li, and Y. Wang, "Blow-up of the solution for higherorder Kirchhoff-type equations with nonlinear dissipation," Central European Journal of Mathematics, vol. 9, no. 3, pp. 686698, 2011.

[21] F. S. Li and J. L. Li, "Global existence and blow-up phenomena for nonlinear divergence form parabolic equations with inhomogeneous Neumann boundary conditions," Journal of Mathematical Analysis and Applications, vol. 385, no. 2, pp. 1005-1014, 2012.

[22] F. Li and Q. Gao, "Blow-up of solution for a nonlinear Petrovsky type equation with memory," Applied Mathematics and Computation, vol. 274, pp. 383-392, 2016.

[23] F. Li and J. Li, "Global existence and blow-up phenomena for p-Laplacian heat equation with inhomogeneous Neumann boundary conditions," Boundary Value Problems, vol. 2014, no. 218, 14 pages, 2014.

[24] F. Sun, L. Liu, and Y. Wu, "Finite time blow-up for a class of parabolic or pseudo-parabolic equations," Computers \&
Mathematics with Applications, vol. 75, no. 10, pp. 3685-3701, 2018.

[25] X. Peng, Y. Shang, and X. Zheng, "Lower bounds for the blowup time to a nonlinear viscoelastic wave equation with strong damping," Applied Mathematics Letters, vol. 76, pp. 66-73, 2018.

[26] X. Du and A. Mao, "Existence and Multiplicity of Nontrivial Solutions for a Class of Semilinear Fractional Schrödinger Equations," Journal of Function Spaces, vol. 2017, Article ID 3793872, 7 pages, 2017.

[27] Q. Feng and F. Meng, "Explicit solutions for space-time fractional partial differential equations in mathematical physics by a new generalized fractional Jacobi elliptic equation-based subequation method," Optik - International Journal for Light and Electron Optics, vol. 127, no. 19, pp. 7450-7458, 2016.

[28] Y. Guo, "Nontrivial solutions for boundary-value problems of nonlinear fractional differential equations," Bulletin of the Korean Mathematical Society, vol. 47, no. 1, pp. 81-87, 2010.

[29] Y. Guo, "Solvability of boundary-value problems for nonlinear fractional differential equations," Ukrainian Mathematical Journal, vol. 62, no. 9, pp. 1409-1419, 2011.

[30] A. Qian, "Sing-changing solutions for nonlinear problems with strong resonance," Electronic Journal of Differential Equations, vol. 2012, no. 17, 8 pages, 2012.

[31] Q. Feng and F. Meng, “Traveling wave solutions for fractional partial differential equations arising in mathematical physics by an improved fractional Jacobi elliptic equation method," Mathematical Methods in the Applied Sciences, vol. 40, no. 10, pp. 3676-3686, 2017.

[32] F. Li, "Limit behavior of the solution to nonlinear viscoelastic Marguerre-von Kármán shallow shell system," Journal of Differential Equations, vol. 249, no. 6, pp. 1241-1257, 2010.

[33] A. Qian, "Infinitely many sign-changing solutions for a Schrödinger equation," Advances in Difference Equations, vol. 2011, no. 39, 6 pages, 2011.

[34] H. Liu and F. Meng, "Interval oscillation criteria for secondorder nonlinear forced differential equations involving variable exponent," Advances in Difference Equations, Paper No. 291, 14 pages, 2016.

[35] K. M. Zhang, "On a sign-changing solution for some fractional differential equations," Boundary Value Problems, vol. 2017, no. 59, 8 pages, 2017.

[36] J. Shao, Z. Zheng, and F. Meng, "Oscillation criteria for fractional differential equations with mixed nonlinearities," Advances in Difference Equations, p. 323, 2013.

[37] R. Xu and F. Meng, "Some new weakly singular integral inequalities and their applications to fractional differential equations," Journal of Inequalities and Applications, vol. 2016, no. 78, 2016.

[38] Y. Wang and J. Jiang, "Existence and nonexistence of positive solutions for the fractional coupled system involving generalized p-Laplacian," Advances in Difference Equations, Paper No. 337, 19 pages, 2017.

[39] D. Lu, C. Yue, and M. Arshad, “Traveling Wave Solutions of Space-Time Fractional Generalized Fifth-Order KdV Equation," Advances in Mathematical Physics, vol. 2017, Article ID 6743276, 6 pages, 2017.

[40] W. Yuan, Y. Li, and J. Lin, "Meromorphic solutions of an auxiliary ordinary differential equation using complex method," Mathematical Methods in the Applied Sciences, vol. 36, no. 13, pp. 1776-1782, 2013

[41] W. J. Yuan, Y. D. Shang, Y. Huang, and H. Wang, "The representation of meromorphic solutions to certain ordinary differential 
equations and its applications," Scientia Sinica Mathematica, vol. 43, no. 6, pp. 563-575, 2013.

[42] W. Yuan, Q. Chen, J. Qi, and Y. Li, “The general traveling wave solutions of the Fisher equation with degree three," Advances in Mathematical Physics, Art. ID 657918, 5 pages, 2013.

[43] Y. Gu, W. Yuan, N. Aminakbari, and Q. Jiang, "Exact Solutions of the Vakhnenko-Parkes Equation with Complex Method," Journal of Function Spaces, vol. 2017, Article ID 6521357, 6 pages, 2017.

[44] G. Jumarie, "Modified Riemann-Liouville derivative and fractional Taylor series of nondifferentiable functions further results," Computers \& Mathematics with Applications, vol. 51, no. 9-10, pp. 1367-1376, 2006.

[45] G. Jumarie, "Table of some basic fractional calculus formulae derived from a modified Riemann-Liouville derivative for nondifferentiable functions," Applied Mathematics Letters, vol. 22, no. 3, pp. 378-385, 2009.

[46] A. E. Eremenko, L. Liao, and T. W. Ng, "Meromorphic solutions of higher order Briot-Bouquet differential equations," Mathematical Proceedings of the Cambridge Philosophical Society, vol. 146, no. 1, pp. 197-206, 2009.

[47] N. A. Kudryashov, "Meromorphic solutions of nonlinear ordinary differential equations," Communications in Nonlinear Science and Numerical Simulation, vol. 15, no. 10, pp. 2778-2790, 2010.

[48] S. Lang, Elliptic Functions, Springer, New York, NY, USA, 2nd edition, 1987.

[49] R. Conte and M. Musette, "Elliptic general analytic solutions," Studies in Applied Mathematics, vol. 123, no. 1, pp. 63-81, 2009. 


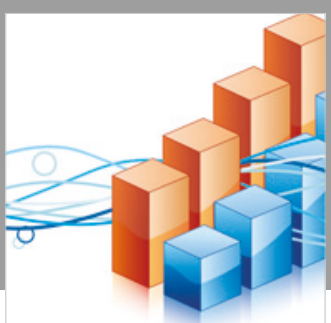

Advances in

Operations Research

\section{-n-m}
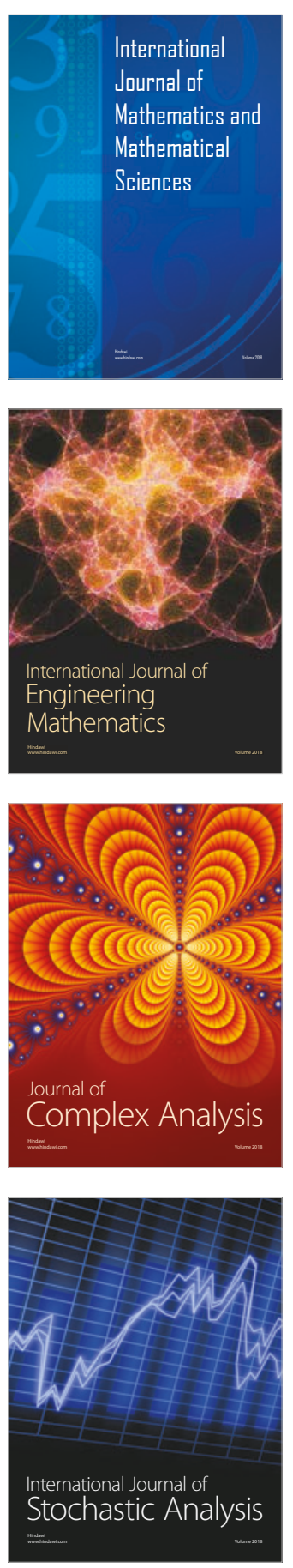
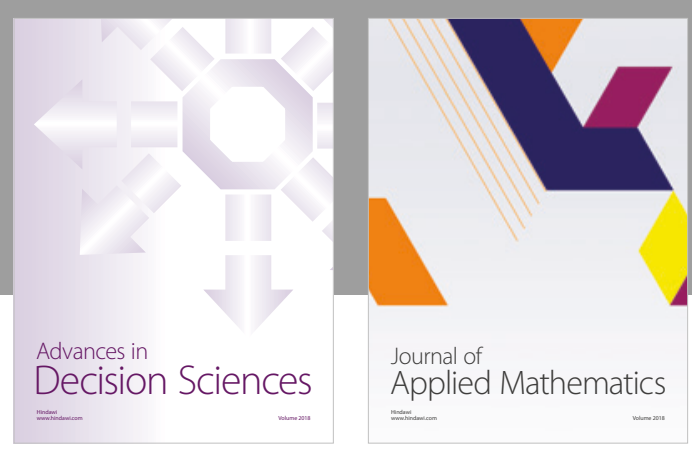

Journal of

Applied Mathematics
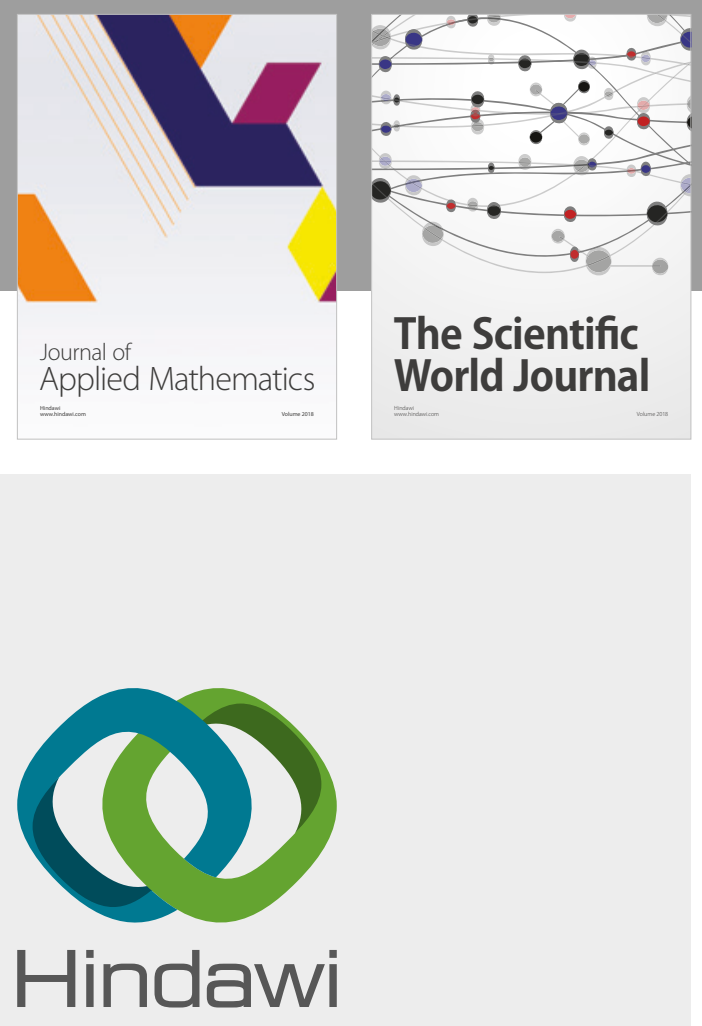

Submit your manuscripts at

www.hindawi.com

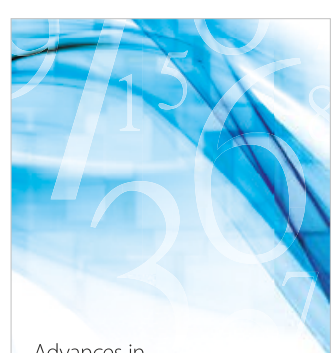

Advances in
Numerical Analysis
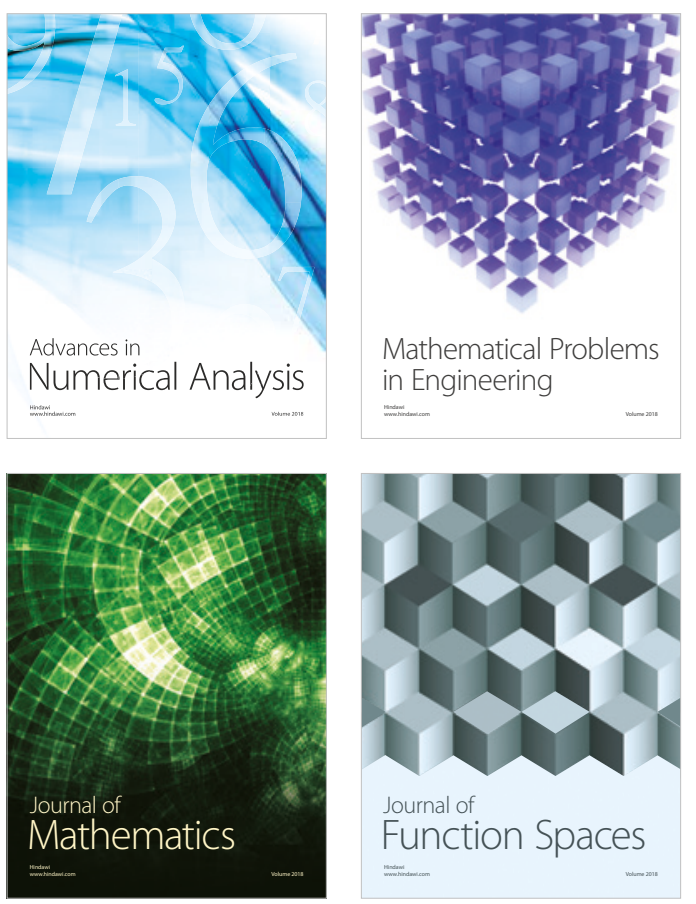

Mathematical Problems in Engineering

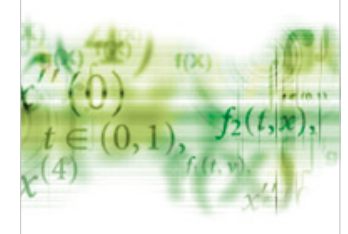

International Journal of

Differential Equations

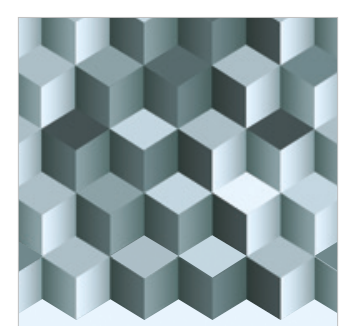

Journal of

Function Spaces

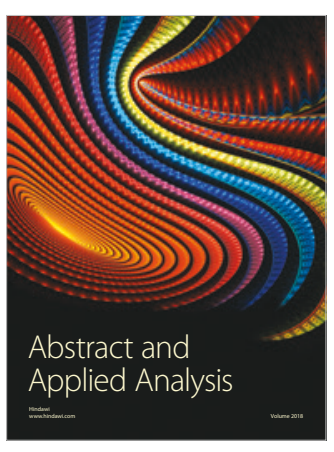

The Scientific

World Journal

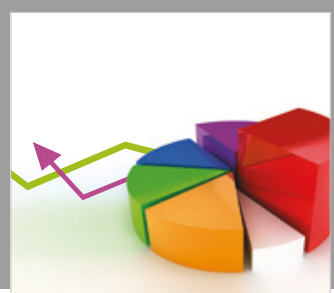

Journal of

Probability and Statistics
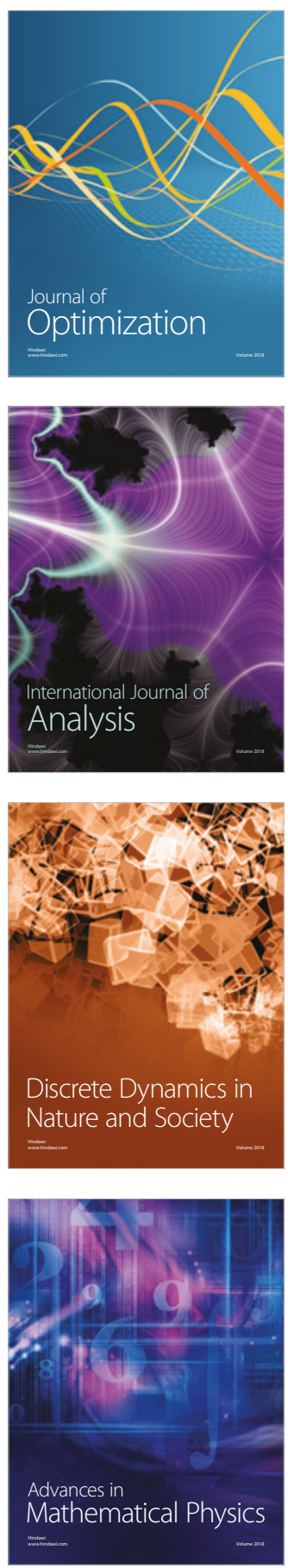